\title{
Design Convenience Stores for Chinese Teenagers
}

\author{
Jia Zhou, Pei-Luen Patrick Rau, Hui Li, Wei Jiang, Bayan Konirbay, Christian \\ Seyfert, Kanta Sribunnak, and Christoph Winkler
}

\author{
Department of Industrial Engineering \\ Tsinghua University, Beijing 100084, P.R. China \\ zhou-jia07@mails.tsinghua.edu.cn, rpl@mail.tsinghua.edu.cn, \\ hli.sunshine@gmail.com, jangvei@gmail.com, bayan_kb@yahoo.com , \\ Christian.Seyfertarwth-aachen.de, blue_karamel@hotmail.com, \\ winkler.christopheymail.com
}

\begin{abstract}
This study aimed to design convenience stores to attract Chinese teenagers. The convenience store's location, product, and image were studied. An observation and an interview were conducted to collect teenagers' requirements. After that, a conceptual design was created and high-fidelity prototype was developed. Twenty middle school students were recruited to evaluate the design concept. The results indicated that convenience stores oriented to teenagers should be located near a school, on the way to the bus stop, as close to the school as possible. Tasty meals, snacks, and self-service food and drink should be used as competing factors. Meanwhile, a positive and modern image is the deciding factor to attract teenagers. Implications for designing convenience stores for Chinese teenagers were discussed.
\end{abstract}

Keywords: Convenience store, Teenagers, User study, Experience-based design.

\section{Introduction}

Convenience store is an important part of our everyday life. It is a place where we can easily get daily necessities we want. In places with high population density such as Tokyo, London, New York or Hong Kong, convenience stores are sprouting from every corner, serving the needs of everyone by selling products ranging from candy, ice cream, sandwiches, magazines, newspapers and even lottery tickets.

In recent years, China has remained the fastest growing convenience store market in the world. Take the capital city Beijing for example. Beijing with a population of 17 million and growing is an ideal location for convenience store business to grow.

Teenagers in China are the trendsetters and are more influenced by the peers. They will buy the product when most of their peers have, even if they really don't need it. $51.3 \%$ of the Chinese teenagers admitted that they are easily be influenced by others [1]. Another distinctive characteristic of Chinese teenagers is that they hunt for novelty and desire to be unique. On the one hand, they have great interests in fashions and try to go with the times; but on the other hand, they still like to be unique. The distinctive characteristics of herd mentality and keeping track of fashions make the 
Chinese teenagers potential customers for the convenience store market in China. However, current Chinese convenience stores are not designed to meet their expectations. There is a great opportunity for Chinese convenience stores to enhance the percentage of teenage customers and to increase their sales volume.

In order to expend the teenagers' market of convenience stores in China, this study focuses on discovering a way to make convenience stores more attractive to Chinese teenagers, and to make convenience stores a trendy and convenient place for them to go. The objective of this study is to analysis requirements of Chinese teenagers and to design a convenience store to meet the requirements of young customers in the age of 12 to 18 years old. Experienced-based design approach was used to capture and understand teenagers' experiences and stories. The proposed design concept enhanced the end-to-end teenager's experience process when visiting convenience stores.

In the remainder of this paper, we first reviewed related literatures. Then we described the methodology used in this study. After that, the conceptual design and the evaluation results were presented. We ended the paper by discussing the results and implications for designing convenience stores for teenagers.

\section{Background Literatures}

Convenience store is a small store or shop that generally sells food, sweets, ice-cream, soft drinks, newspapers and magazines. Convenience stores often locate alongside the busy roads, in densely-populated urban neighborhoods, near gas stations, or near railway station. Some convenience stores open for 24 hours.

When considering the design of a convenience store, location might be one of the most important decisions. Location selection plays a very important role. It is particularly crucial for the convenience store with its dependence on high customer flows and low value transactions [2]. Many studies have examined different methods to design convenience store locations. For example, Kuo, Chi and Kao [3] developed a decision support system for locating a new convenience store. Their proposed system consists of four components: (a) hierarchical structure development for fuzzy analytic hierarchy process; (b) weights determination, (c) data collection, and (d) decision making. The results showed that the proposed system is able to provide more accurate result than the regression model.

Store image also serves an important role for the success of a convenience store. In retail store design, color is used to attract or to draw customer's attention. It is also used to project an image or to create a desired atmosphere for a store. Color can produce certain autonomic biological reactions, create certain emotional responses, and obtain attentions. Bellizzi, Crowley, and Hasty [4] evaluated the effects of color in store design. They found out that color can physically attract shoppers toward a retail display and have certain perceptual qualities that affect store and merchandise image.

Previous studies on the design of convenience stores mainly focused on the general design. Very few studies have been done to collect teenagers' requirements and design convenience stores for them. Teenagers have their special needs for the goods supplied in convenience stores. They are highly interested in PC-Games, fashions and comics. Stars, movies, comics, and games are hot topics in teenagers' web forums. Thus, in this study, we aimed to design a convenience store for Chinese teenagers. 


\section{Methodology}

The overall goal for this study was to design convenience stores to attract Chinese teenagers. In this study, we focused on the design of location, products, and image aspects of the convenience stores. In more detailed, we aimed to study the following three questions.

1. What is the optimal location for an exemplary convenience store to attract teenagers?

2. What products are most important for Chinese teenagers and fit into the convenience store concept?

3. How to improve the image of convenience store to attract the teenagers?

In order to answer these questions, user-centered design approach was used. We first conducted an observation to learn about teenagers' activities after school. After that, we interviewed four teenagers to specify their requirements. Based on the findings from user study, a conceptual design was created and twenty teenagers were recruited to evaluate the design.

\subsection{Observation}

The aim of the observation was to learn more about teenagers' activities after school. Direct observation was conducted at one middle school in Beijing. The observation was performed in the afternoon right after the classes were over, when about one hundred students left the campus to go home.

It was observed that $46.8 \%$ of the students took bus to go home, $28.0 \%$ rode bike, $14.0 \%$ walked home, and $11.2 \%$ were picked up. After school, many students headed to kiosks. High demands of buying food or drinks after school were observed, but there were only kiosks near school, which can not satisfy their requirements. Thus, how to reach them after school is an important aspect when designing convenience stores to attract Chinese young customers.

\subsection{Interview}

In addition to observation, a structured interview with both open and close ended questions was conducted. The purpose of the interview was to specify teenagers' requirements on products and image aspect of convenience stores. Two boys and two girls aged from 14 to 16 studying at one school in Beijing were interviewed. During the interview, we encouraged teenagers to tell their experiences and stories when they visit convenience stores.

For the products aspect, the interview showed that food and snacks were most attractive for teenagers to go to convenience stores. They mostly go to convenience stores after school to buy food, mainly for the taste of it, instead of hunger. The food bought there was mainly eaten on the way back home. Girls were interested in a large variety of food and were interested in healthy food such as fruits. They liked the general idea of "do-it-yourself" very much regarding the food or drink preparation. They also concerned the hygienic standards a lot. In contrast, although the boys being asked about the self-service concept accepted the idea, they were not that 
enthusiastically as the girls. School supplies being offered in convenience stores were regarded as too expansive by the boys; however for the girls, they were interested in school supplies fitting to their needs of pretty objects. Girls additionally demanded for little things such as toys and gifts or festival-related special offers very much.

For the image aspect, the general preference of western style in the appearance of the store was very clear, and the preferred style was often described as "modern". Boys as well as girls opted for a clear and bright decoration. They suggested colors as "bright" and "simple". They preferred low volume and easy background music. The idea of the adaption of the decoration to seasonal festivals was very well liked especially by the girls. When talking about the shop assistant, the most important point was the efficiency. Friendly and helpful were major requirements for their ideal shop assistant as well, whereas age and gender played a minor role.

In conclusion, we can see that the main focus concerning the products should be laid on the food aspect as this attracts Chinese teenagers most. A self-service concept for food and drink would meet teenagers' demands best. Very important is the aspect of hygiene which should be made clear by a clean and tidy store especially in the food area. Chinese style fast food with good taste can be provided to attract the teenagers. School supplies can also be provided at stores. When arranging seating area, it is better to be located at the window front, and be separated from the crowded areas, e.g. the checkout and the food preparation area. For the environment in convenience stores, a bright and modern color should be selected, and gentle background music at low volume can be played. Before upcoming festivals, the decoration and product offer can be slightly adapted. Shop assistants need to wear some kind of uniform, work at high efficiency, and be friendly and helpful. The health aspect is very important to teenagers. Thus, the store should be able to combine efficiency with the air of a young, modern and health-conscious store. It can be found in the products as well as in the image of the store.

\section{Conceptual Design and Evaluation}

Based on the results of the user study, a conceptual design was created and evaluated. The conceptual design focused on the design of location, products, and the image aspects of convenience stores for Chinese teenagers.

\subsection{Location}

Ideally, the convenience stores should locate as close as possible to the school. However, there are usually many factors restrict the location of convenience stores to be near school. Thus, we want to exam how location influences teenagers' preference.

We consider the possible location of convenience stores along the road from school to bus station and further away. Besides, the situation of crossings was also considered. Figure 1 shows the possible locations of six convenience stores along the road from school to bus stop and further away. Convenience store A is close to school, convenience store B is 50 meter away from the school, and so forth, convenience store $\mathrm{F}$ is 250 meter away from school. Bus station is shown by symbol $\mathrm{H}$ in the figure as an example. Figure 1(a) illustrates the situation of no crossings, and figure 1(b) illustrates the situation of having crossings along the road. 


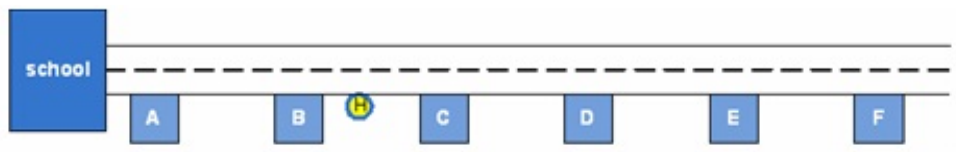

(a)

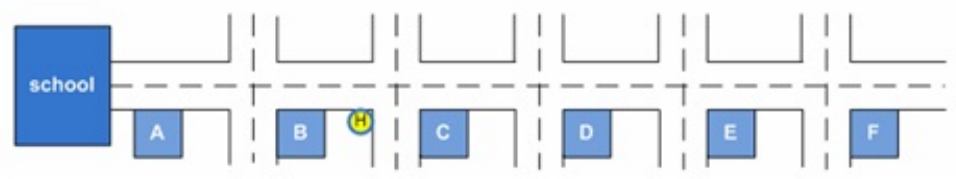

(b)

Fig. 1. Examine the influence of location on visiting convenience stores

Twenty students from one middle school in Beijing were asked for their preference on visiting the convenience stores if the store locates at the possible twelve locations with and without crossing conditions. The result is shown in Figure 2. Positive scores mean that teenagers would like to visit the convenience store, while the negative scores mean that they would not go. The higher the score, the more likely they visit the convenience stores.

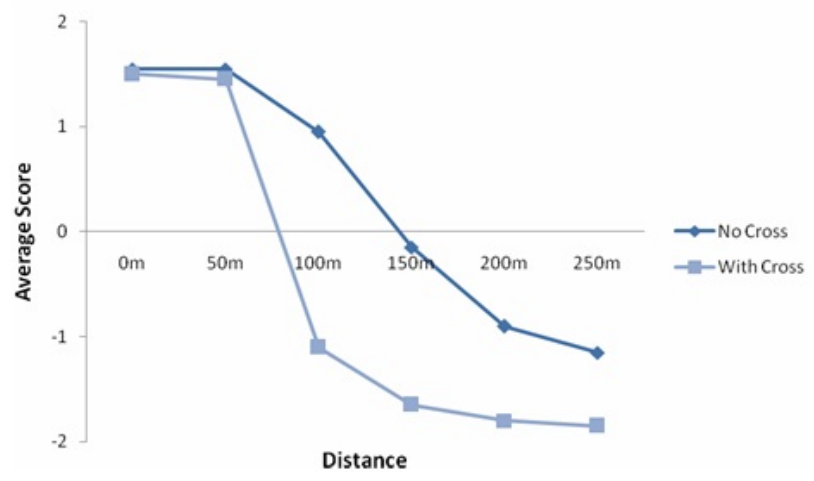

Fig. 2. Results of distance and crossings on visiting convenience stores

No obvious preference was shown as long as the convenience store is on the way from the school to the bus stop. But teenagers showed reluctance to take more walk to go to convenience stores especially if there are crossings they have to cover. Preferences decreases exponentially with distance increasing from school to possible convenience stores, and especially for having crossing's condition.

\subsection{Products and Images}

The next step was to design the products and image for the convenience store. Products can be divided into four major product groups: food, drink and fruit; 
magazines and comics; school supplies; and additional products and services. The following design concepts were developed for different product groups.

- Food, drink, and fruit

- Guideline: "Tasty and healthy!"

- Mostly Chinese food

- Self-service food

- Self-service drink

- Fruit cocktails

- Soft drinks

- Single fruits

- Cups of sliced fruit

- Magazines and comics

- Broad, offer gender-specific comic and magazine

- Increasing sales volume allows lower prices and competition with bookstores

- School supplies

- Basic equipment and consumables

- Additional products and services (e.g., cinema-related services, seasonal products, knickknacks, etc.)

- Movie trailer

- Cinema ticket booking service

- Seasonal and festival related goods

- Cute and funny things and presents

At last, the convenience store design was completed with the definition of the image aspect. The store color is green, since it corresponds with youth. The shop motto is "cinema", because the participants mentioned in the interview that this is their popular activity for spare time. Therefore, film posters will be put on the wall and movie tickets can be sold in convenience stores. The followings are the key design concepts for image aspect.

- Different shades of green color

- Seating possibilities

- Indirect lighting

- Background music

- Young and fashionable shopkeepers

In order to evaluate the design, a high-fidelity prototype was developed. Figure 3 shows the 3D overview of the store. Figure 4 shows the detailed design for the store, for example, the food, the magazines and comics, and the seating area in the store.

Twenty students from one middle school in Beijing were recruited to evaluate the design concept. The results showed that the design had a positive attraction to young customers. Appealing and tasty food was the most attractive product for Chinese teenagers. Self-service ideas were also very attractive to teenagers, but this has to be kept simple and hygienic. Besides, teenagers rated high scores for background music 


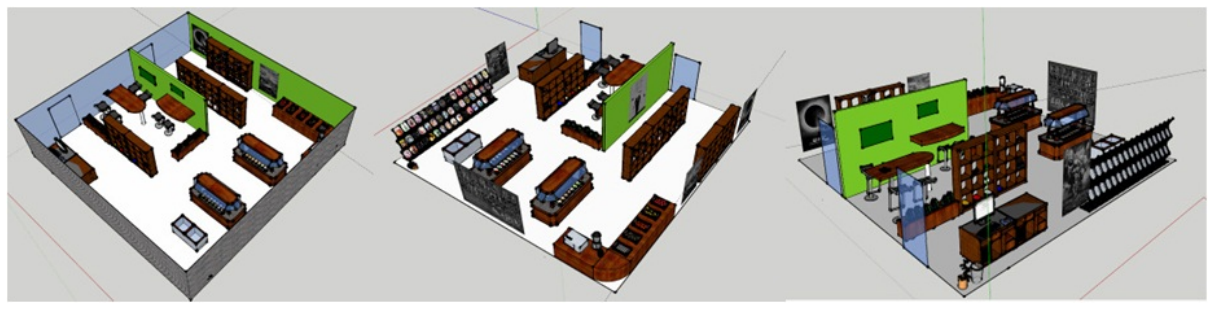

Fig. 3. 3D overview of the convenience store
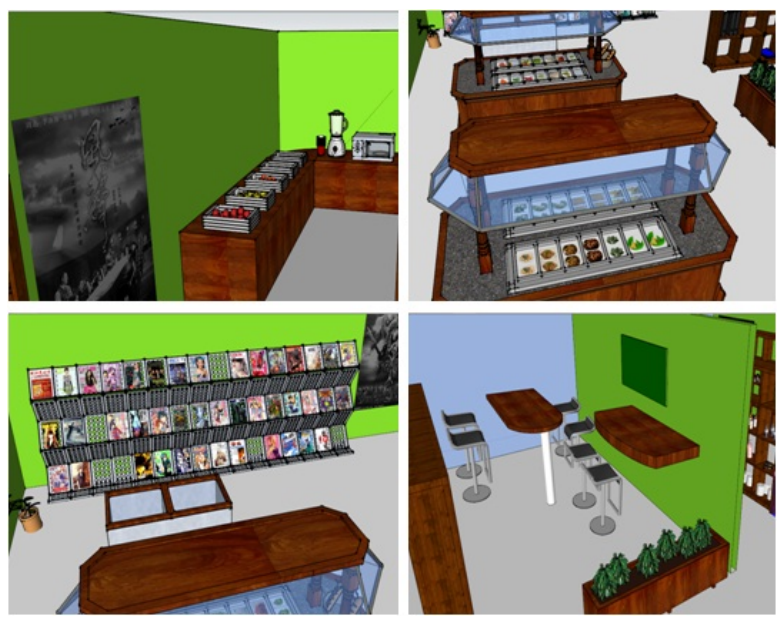

Fig. 4. Detailed design of the convenience store

and color of the store, but they cared less about the lighting. Cinema ticket services were also accepted and welcomed by teenagers.

\section{Conclusion}

This study tried to design convenience stores for Chinese teenagers. The location, products, and image aspects of convenience stores were studied. Experience-based design approach was used to understand teenagers' experiences when visiting convenience stores. Customer experience based solutions were transformed to design concepts and were evaluated by high-fidelity prototypes.

The results indicated that a convenience store oriented to teenagers should be located near a school, on the way to the bus stop, as close to the school as possible. In addition, many improvements on the products and image aspects of convenience stores to attract young customers were also provided. The main motivation for Chinese teenagers to visit a convenience store is the food being provided. Tasty meals and snacks will therefore attract customers and should be used as a competing factor. The self-service model for food and drink is highly recommended. For store's image, a positive and modern image can be the deciding factor to compete in the market. 
In conclusion, location, products and image are important aspects when designing convenience stores for teenagers. The results of this study showed that our design concept was very well accepted by Chinese teenagers. But there are still some other factors, for example the price factor hinders the popularity of convenience stores to Chinese teenagers. To be able to compete in this young market segment, new ground has to be broken to lower the price level in teenager oriented convenience stores.

Acknowledgement. This research was funded by Asia Research center: "2009 young researcher grant from Asia Research Center in Tsinghua University".

\section{References}

1. Cheng, S.: Minor consumers: Understand Chinese School Students Today. China Light Industry Press, Beijing (2004)

2. Kirby, D.A.: Convenience Stores: The polarisation of British retailing. International Journal of Retail \& Distribution Management 14, 7-12 (1986)

3. Kuo, R.J., Chi, S.C., Kao, S.S.: A decision support system for selecting convenience store location through integration of fuzzy AHP and artificial neural network. Computers in Industry 47, 199-214 (2002)

4. Bellizzi, J.A., Crowley, A.E., Hasty, R.W.: The Effects of Color in Store Design. Journal of Retailing 59, 21-45 (1983) 